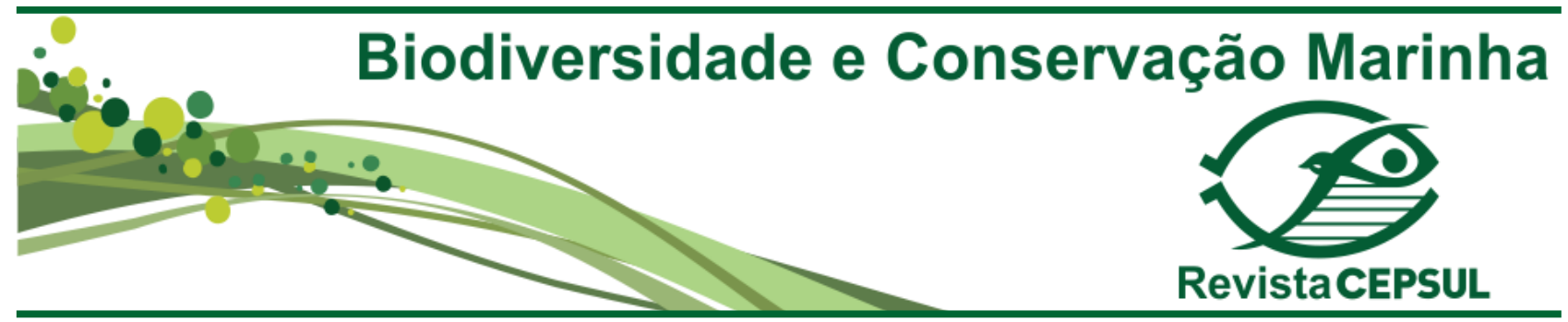

ARTIGO CIENTÍFICO

\title{
Avaliação quali-quantitativa de algas marinhas macrófitas ocorrentes na Praia da Vigia, Garopaba/SC
}

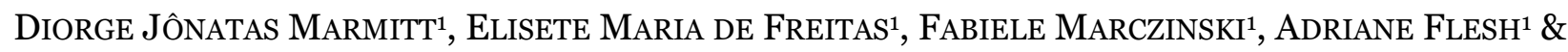 \\ ÉDINA APARECIDA REIS BLASI ${ }^{1}$
}

${ }^{1}$ Centro Universitário UNIVATES, Rua Avelino Tallini, 171, Bairro Universitário CEP - 959oo-ooo, Lajeado, RS, Brasil, diorgemarmitt@yahoo.com.br, elicauf@univates.br,fabim87@yahoo.com.br,adriflesch@hotmail.com, erblasi@yahoo.com.br

Resumo. O estudo teve por objetivo, identificar espécies e conhecer a estrutura da comunidade de algas da Praia da Vigia, Garopaba, Santa Catarina, pertencentes aos filos Rhodophyta (algas vermelhas), Phaeophyta (algas pardas) e Chlorophyta (algas verdes). Foram distribuídas 100 unidades amostrais de $0,25 \mathrm{~m}^{2}$ de modo aleatório em nove pontos ao longo da praia, considerando locais de submersão contínua e imersão temporária. Foram registradas 15 espécies. O filo Rhodophyta foi representado por sete espécies, seguido por Chlorophyta e Phaeophyta, com cinco e três espécies cada. Os índices de diversidade estimados pelo Shannon ( $\left.\mathrm{H}^{\prime}\right)$ e equabilidade de Pielou $(\mathrm{J})$ foram, respectivamente, 1,95 nats.ind.-1 e 0,72. Os resultados obtidos indicam que a espécie de maior valor de importância foi Ulva lactuca, seguida por Chaetomorpha antennina e Sargassum cymosum. Além dos baixos valores de diversidade, houve pouca diferença na estrutura da comunidade e a distribuição das espécies entre os locais de inundação constante e temporária foi pequena.

Palavras-chave: comunidade de algas; algas bentônicas; litoral catarinense; diversidade ficológica.

\begin{abstract}
Qualitative and quantitative evaluation of macrophytes seaweeds occurring in the Vigia Beach, Garopaba/SC. The study aimed to identify species and to know the structure of the algae community of the Praia da Vigia, Garopaba, Santa Catarina, belonging to the phyla Rhodophyta (red algae), Phaeophyta (brown algae) and Chlorophyta (green algae) . There were distributed 100 sample units of $0.25 \mathrm{~m}^{2}$ at random in nine points along the beach, considering places of continuous submersion and temporary immersion. Were registered 15 species. The phylum Rhodophyta was composed for seven species, followed by Chlorophyta and Phaeophyta, with five and three species each. The diversity indices estimated by Shannon ( $\left.\mathrm{H}^{\prime}\right)$ and equability of Pielou $(\mathrm{J})$ were, respectively, 1,95 nats.ind.1 and 0,72 . The results indicate that the species of greatest importance value was Ulva lactuca, followed by Chaetomorpha antennina and Sargassum cymosum. In addition to the
\end{abstract}


low diversity values, there was a little difference in community structure and the distribution of species between the place constant flood and place temporary flood was small.

Keywords: benthonic algae; Santa Catarina coast; ficologic diversity.

\section{Introdução}

As algas estão entre os primeiros organismos a ocupar o planeta e caracterizam-se por serem organismos fotossintetizantes, predominantemente de ambiente aquático (Raven et al., 2007).

Dentre as algas macroscópicas destacam-se os filos Rodophyta, Phaeophyta e Clorophyta. As Rodophyta (algas vermelhas), em sua maioria, ocorrem em ambientes marinhos geralmente no bentos, predominando nos mares tropicais. Elas produzem ficocolóides, principalmente ágar e carragenano, utilizados em meios de culturas, nas indústrias alimentícias, farmacêuticas, de cosméticos e de tintas (Littler et al., 1985).

As Phaeophyta (algas pardas) são, em sua grande maioria, marinhas (exceto por três gêneros raros de água doce) e podem, nos mares tropicais, acumular grande quantidade de biomassa. Produzem os alginatos, utilizados como agentes geleificantes, emulsificantes e estabilizantes nas indústrias alimentícias (como fabricação de sorvetes), têxteis, de cosméticos, farmacêuticas e na fabricação de cerveja (Raven et al., 2007).

Já as Clorophyta (algas verdes), embora ocorram em ambientes marinhos, predominam em águas continentais. Também podem habitar troncos de árvores, solos, superfícies de neve e viver em simbiose com fungos (liquens), protozoários, hidras, esponjas e outros animais. Produzem diversos compostos como terpenóides (Blunt et al., 2005), clorofilas a e b, xantófilas (principalmente, a luteína) e carotenos (principalmente, o $\beta$-caroteno) (Naguib, 2000), os quais são de grande interesse para as indústrias de alimentos, cosméticos e farmacêutica (Raven et al., 2007).
De acordo com Judd et al. (2009), as algas formam a base da grande maioria das cadeias tróficas aquáticas e contribuem com a produção da maior parte do oxigênio disponível na atmosfera. Também servem de berçário e refúgio para diversas espécies de peixes, crustáceos, moluscos, mamíferos e vários outros grupos de animais. Portanto, desempenham papel ecológico fundamental na manutenção dos ecossistemas (Oliveira et al., 2009). São responsáveis pela fixação de nitrogênio, podendo reduzir ou até evitar o uso de fertilizantes na agricultura e ainda, podem ser usadas na correção da acidez de solos (pH) (Raven et al., 2007).

Apesar das comunidades algáceas apresentarem considerável diversidade de espécies, o conhecimento sobre a sua estrutura ainda é escasso, o que dificulta uma maior compreensão sobre esse ecossistema (Pereira, 2002). Estudos relacionados à caracterização de comunidades de macroalgas marinhas são recentes no litoral brasileiro, a maioria trata da caracterização de comunidades algáceas do infralitoral, com bancos de rodolitos (Amado-Filho et al., 2007, 2010; Riul et al., 2009) e formações recifais (Villaça \& Pitombo 1997; Horta et al., 2001; Figueiredo et al., 2004; Amado-Filho et al., 2007, 2010; Cocentino et al., 2010).

Dentre os estudos que enfocam estruturas de comunidades de macroalgas do litoral brasileiro no mediolitoral destacam-se Muñoz \& Pereira (1997), Barbosa et al. (2008) e Villaça et al. (2010). Ghilardi et al. (2008) afirmam que trabalhos quantitativos das zonas de entremarés no Brasil se restringem principalmente à zona compreendida entre o nível da maré baixa e da ação das ondas na maré alta. No Brasil, em ambientes submersos, estudos a partir de tran- 
secções em fundos rochosos não são comuns.

Amaral \& Jablonski (2005) afirmaram que os estudos existentes na costa marítima do estado de Santa Catarina eram insuficientes, pois o número de espécies registrado era bem inferior ao conhecido mundialmente. No entanto, para Seeliger et al. (1998), as temperaturas baixas no sul do Brasil e a diminuição dos costões rochosos na costa marítima do estado de Santa Catarina representam barreiras na dispersão dos organismos marinhos que dependem de substrato duro para se estabelecerem. Walker \& Kendrick (1998) já afirmavam que a diversidade biológica na zona de entremarés estava sendo reduzida em consequência das mudanças e interferências antrópicas provenientes dos mais diversos fatores, dos quais sobressaem a exploração das espécies com valor econômico, a introdução de espécies exóticas, poluição, fragmentação e destruição de hábitats.

Neste sentido, é de extrema importância conhecer as espécies existentes de algas ma- rinhas macroscópicas sésseis e compreender possíveis mudanças ambientais, fornecendo atributos que visem medidas de proteção à biodiversidade e ao patrimônio genético (Palumbi, 2001). Assim, o objetivo do estudo foi identificar espécies e conhecer a estrutura da comunidade de algas da Praia da Vigia, Garopaba, Santa Catarina, pertencentes aos filos Rhodophyta (algas vermelhas), Phaeophyta (algas pardas) e Chlorophyta (algas verdes).

\section{Material e métodos}

\section{Área de estudo}

Garopaba, situada na região sul de Santa Catarina, é uma enseada que se estende da Ponta da Faísca ou Gamboa até a Ponta do Ouvidor, banhada pelo Oceano Atlântico (Prefeitura Municipal de Garopaba, 2013) (Figura 1). As planícies largas e contínuas prevalecentes a sul dão lugar, ao norte, à paisagem dominada por baías, pequenas lagunas, ilhas e praias recortadas pelo embasamento protero-

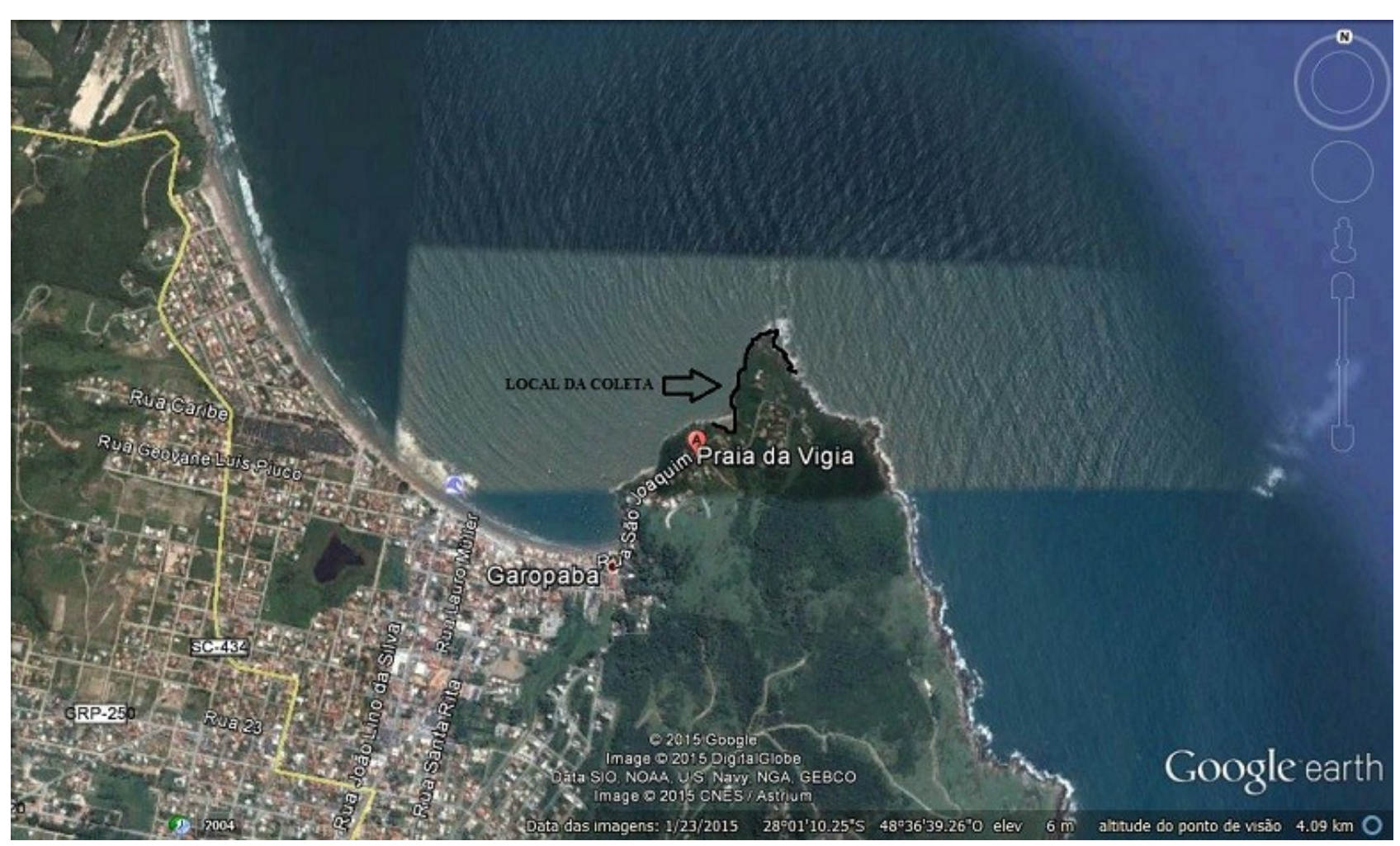

Figura 1. Mapa da região de Garopaba, delimitando a área de estudo na Praia da Vigia. Fonte: Google Earth. 


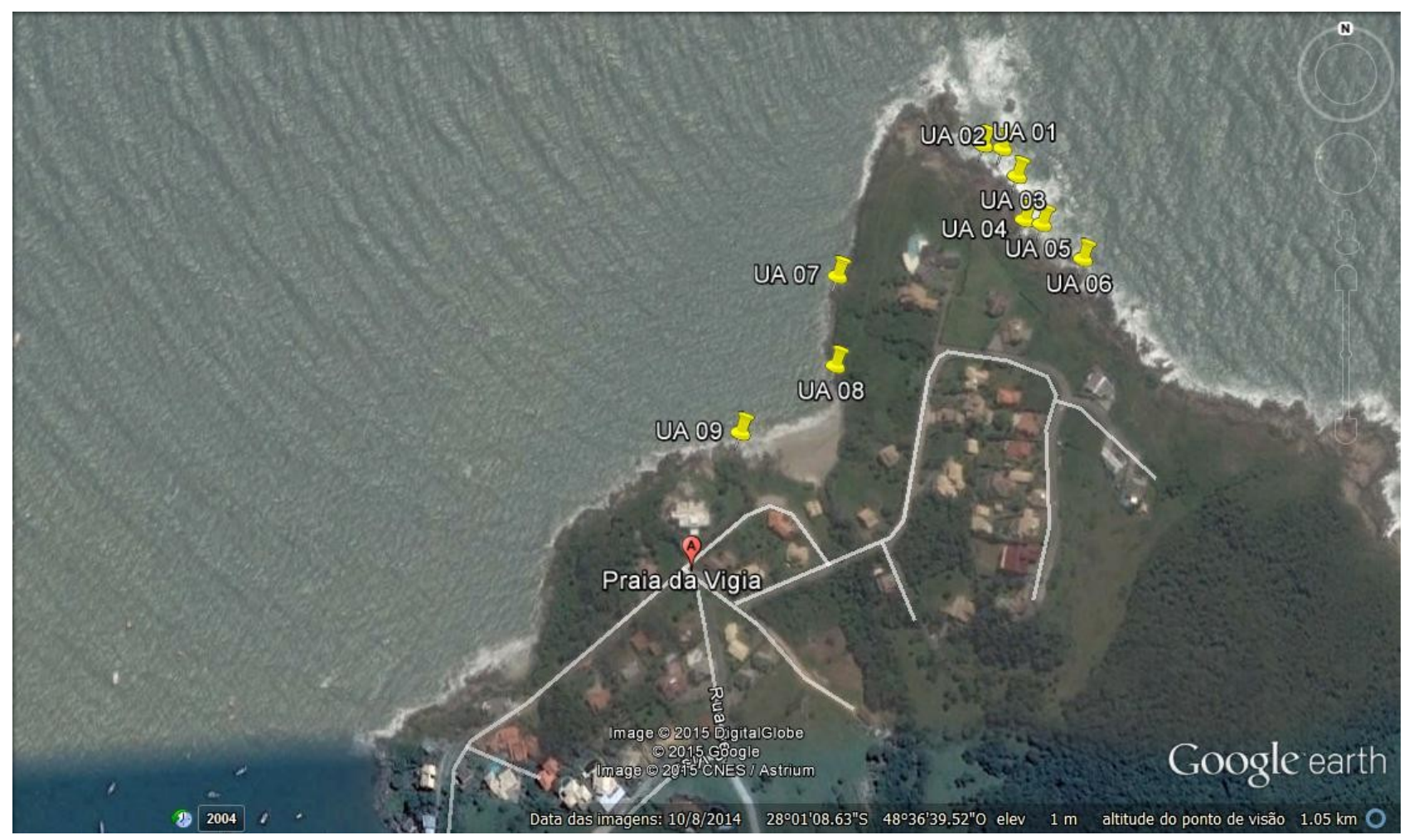

Figura 2. Imagem de satélite da localização dos locais de distribuição das unidades amostrais na Praia da Vigia, Garopaba, Santa Catarina. Fonte: Google Earth.

zóico (Martin et al., 1988).

Conforme Martin et al. (1988), no município de Garopaba, são reconhecidas quatro unidades geomorfológicas: areias marinhas litorâneas, sedimentos argilosos e arenosos de lagunas e baías, sedimentos argilo-arenosos flúvio-lagunares e sedimentos continentais mal selecionados. Destas, as três primeiras são encontradas na área de estudo.

\section{Amostragem da comunidade de algas}

O estudo foi realizado com amostragem pontual nos dias 03 e 04 de maio de 2013, em uma saída a campo durante a disciplina de Sistemática e Evolução de Criptógamas do curso de Ciências Biológicas do Centro Universitário UNIVATES.

Foram distribuídas 100 unidades amostrais de $0,25 \mathrm{~m}^{2}$ em nove pontos ao longo da costa sul da Praia da Vigia (Figura 2), de modo aleatório na zona de quebra marinha, analisando três tipos de substratos ao longo da costa, areia, rocha não fragmentada e rocha fragmentada. Do total de unidades amostrais, 34 foram distribuídas em locais onde as algas permaneciam submersas continuamente e 66 onde as algas ficavam imersas pelo movimento da água durante as ondas e na maré alta Em cada unidade amostral foram identificadas as espécies existentes, pertencentes aos filos Rhodophyta (algas vermelhas), Phaeophyta (algas pardas) e Chlorophyta (algas verdes). Para cada uma das espécies amostradas foi estimado, visualmente, o percentual de cobertura de cada uma.

Durante a amostragem foi realizada a coleta de um exemplar de cada espécie. As coletas foram acondicionadas em sacos plásticos com álcool 70\%, devidamente etiquetados para identificação em laboratório com o uso de microscópio binocular e bibliografias específicas (Baptista, 1977; Nassar, 2012).

A partir de dados de campo, foram definidos os parâmetros fitossociológicos de frequência (F) e cobertura (C), absolutas e relativas, e o índice de valor de importância (IVI). A 
cobertura absoluta da espécie (CAi) foi obtida a partir da soma dos valores obtidos pela espécie em todas as unidades amostrais em que apareceu. A cobertura relativa de cada espécie foi obtida pela fórmula $\mathrm{CRi}=\left(\mathrm{CAi} / \sum \mathrm{CA}\right) / 100$, sendo $\Sigma$ CA o somatório da cobertura absoluta de todas as espécies. Os valores de frequência absoluta (FAi) e relativa (FRi) de cada uma das espécies foram obtidos, respectivamente, através da aplicação das fórmulas $\mathrm{FAi}=(\mathrm{NUAi} /$ NUAt).100 e FRi $=(\mathrm{FAi} / \Sigma \mathrm{FA}) .100$, onde NUAi corresponde ao número de unidades amostrais em que a espécie apareceu, NUAt corresponde ao total de unidades amostrais estabelecidas na área de estudos e, $\Sigma$ FA é o somatório dos valores de FA de todas as espécies. Os valores de IVI de cada uma das espécies foram obtidos através do uso da fórmula IVI=(CRi+FRi $) / 2$ (Müeller-Dombois \& Ellemberg, 1974). O IVI demonstra a importância ecológica das espécies de uma determinada comunidade, quando comparados aos valores obtidos pelas espécies nela existentes.

A diversidade foi estimada através do índice de Shannon ( $\left.\mathrm{H}^{\prime}\right)$ e a equabilidade pelo índice de Pielou ( $J$ '), aplicando-se as fórmulas $\mathrm{H}^{\prime}=-\left\{\sum[\right.$ pi.LN(pi)] $\}$ e $J=\mathrm{H}^{\prime} / \mathrm{LN}(\mathrm{S})$, Onde S corresponde ao número de espécies, pi à proporção da cobertura encontrada na espécie i em relação ao valor total de cobertura $(\mathrm{pi}=\mathrm{ni} / \mathrm{N})$, ni ao valor de cobertura de toda a amostragem de cada espécie na amostra e $\mathrm{N}$, à cobertura total da amostragem.

O valor do pi foi obtido pela divisão do valor de cobertura absoluta de cada espécie pelo $\Sigma$ CA. E ainda, o LN corresponde ao logaritmo natural e $\mathrm{S}$, ao valor da riqueza (número de espécies amostradas) (Hammer, 2001).

\section{RESULTADOS}

Foram identificadas 15 espécies, das quais sete eram do filo Rodophyta, o mais numeroso, seguido pelos filos Chlorophyta, com cinco espécies, e Phaeophyta com três espécies (Tabela 1). O filo Rodophyta também foi representado por mais famílias (cinco), seguido por Chlorophyta e Phaeophyta com quatro e três

Tabela 1. Lista de espécies com os respectivos filos e famílias registradas na Praia da Vigia, Garopaba, Santa Catarina, no outono de 2013.

\begin{tabular}{lll}
\hline \multicolumn{1}{c}{ Filo } & \multicolumn{1}{c}{ Família } & \multicolumn{1}{c}{ Espécie } \\
\hline \multirow{3}{*}{ Chlorophyta } & Cladophoraceae & Chaetomorpha antennina (Bory de Saint-Vincent) Kützing \\
& Ulvaceae & Codium decorticatum (Woodw.) M.A.Howe \\
& Ulva fasciata Delile \\
& Vaucheriaceae & Vaucheria longicaulis Hoppaugh \\
\hline \multirow{3}{*}{ Phaeophyta } & Chordariaceae & Levringia brasiliensis (Mont.) A.B.Joly \\
& Sargassaceae & Sargassum cymosum C. Agardh \\
& Scytosiphonaceae & Colpomenia cinuosa (Mert. ex Roth) Derbès \& Solier \\
\hline \multirow{5}{*}{ Rhodophyta } & Arthrocardia flabellata (Kütz.) Manza \\
& Cystocloniaceae & Arthrocardia gardineri Manza \\
& Gelidiaceae & Gelidium floridanum W.R.Taylor \\
& Halymeniaceae & Grateloupia filicina (J.V.Lamour.) C.Agardh \\
& Pterocladiaceae & Pterocladia capillacea (S.G. Gmelin) Bornet \\
\hline
\end{tabular}


famílias respectivamente. Ficou claro o predomínio das algas pertencentes ao filo Rodophyta, representado por $41,66 \%$ das famílias e por $46,66 \%$ do total de espécies amostradas. Dentre as famílias, Corallinaceae foi a mais numerosa seguida por Ulvaceae.

Do total de espécies amostradas no levantamento, 13 foram registradas em locais parcialmente inundados e 11 nos locais permanentemente inundados. Hypnea spinella e Levringia brasiliensis não foram registradas nos locais parcialmente inundados e, Arthrocardia flabellata, Codium decorticatum, Gelidium floridanum e Jania rubens não foram registradas nos locais de constante inundação.

O levantamento fitossociológico indicou a predominância da espécie Ulva lactuca, popularmente conhecida por alface do mar, registrada em 50 unidades amostrais e atingindo cobertura relativa de $37,1 \%$. A espécie Chaetomorpha antennina foi a segunda espécie mais abundante, estando presente em 24 unidades amostrais. Esta espécie, pertencente à família Cladophoraceae, é utilizada para remoção de nutrientes, especialmente nitratos, em estágios que há uma massa significativa, partes são removidas, levando os nutrientes absorvidos para fora do sistema (Baptista, 1977).

Ulva lactuca, conhecida como alface-do -mar, foi predominante na costa da praia da Vigia, independente de estar ou não constantemente imersa (Tabela 2), porém houve uma ligeira redução no percentual de cobertura quando em locais de constante inundação em relação aos locais parcialmente inundados. Sendo que nos locais parcialmente inundados, U. lactuca foi sucedida por Chaetomorpha antennina e Sargassum cymosum, e nos locais sob constante inundação, a alface do mar foi seguida por Arthrocardia gardineri e C. antennina.

Considerando toda a amostragem, o índice de Shannon (H') foi de 1,95 nats.ind. ${ }^{-1} \mathrm{e}$ 0,72 para a equabilidade de Pielou (J). Estes valores variaram pouco ao considerar os locais parcialmente inundados $\left(\mathrm{H}^{\prime}=1,83\right.$ nats.ind..$^{-1} \mathrm{e}$ $\mathrm{J}=0,71)$ e constantemente inundados $\left(\mathrm{H}^{\prime}=1,94\right.$ nats.ind. ${ }^{-1}$ e $J=0,81$ ).

\section{DISCUSSÃo}

Resultados similares aos encontrados na costa de Garopaba, também foram descritos por Nunes et al. (1999) no levantamento realizado no município de Ilhéus no estado da Bahia, onde o táxon Rhodophyta foi o mais representativo com 40 espécies e 15 famílias, seguido de Chlorophyta, com 22 espécies em nove famílias, e por Phaeophyta com 15 espécies em quatro famílias. Os resultados também podem ser correlacionados aos descritos por Baptista (1977), em seu estudo sobre flora marinha da costa de Torres, RS, onde também foi registrada maior abundância para o filo Rodophyta.

Levantamentos taxonômicos realizados no mangue do Itacorubi, localizado em Florianópolis, SC, com algas marinhas bentônicas dos filos Chlorophyta (Hadlich, 1984) e Rodophyta (Hadlich \& Bouzon, 1985), mostraram que o filo Rodophyta foi representado por mais espécies do que o filo Chlorophyta, assim como registrado no presente estudo.

A dominância por parte de poucas espécies e a consequente elevação da porcentagem de espécies menos abundantes é comum dentro de uma comunidade (Odum, 1988; Ricklefs, 2003). Entretanto, o resultado do índice de equabilidade próximo de 1,o sugere que a comunidade de macroalgas da Praia da Vigia em Garopaba é homogênea, havendo dominância de poucas espécies.

Tendo em vista os valores do índice de Shannon (H') $\left(1,95\right.$ nats.ind. $\left.^{-1}\right)$ e da equabilidade de Pielou (J) (0,72), é possível estabelecer comparações de diversidade, como por exemplo, com o estudo realizado por Macedo et al. (2009), que avaliaram sete táxons ocorrentes no litoral baiano, sendo quatro da divisão Chlorophyta e três da divisão Rhodophyta. O índice de diversidade das espécies foi de $\mathrm{H}^{\prime}=0,72$ e o 


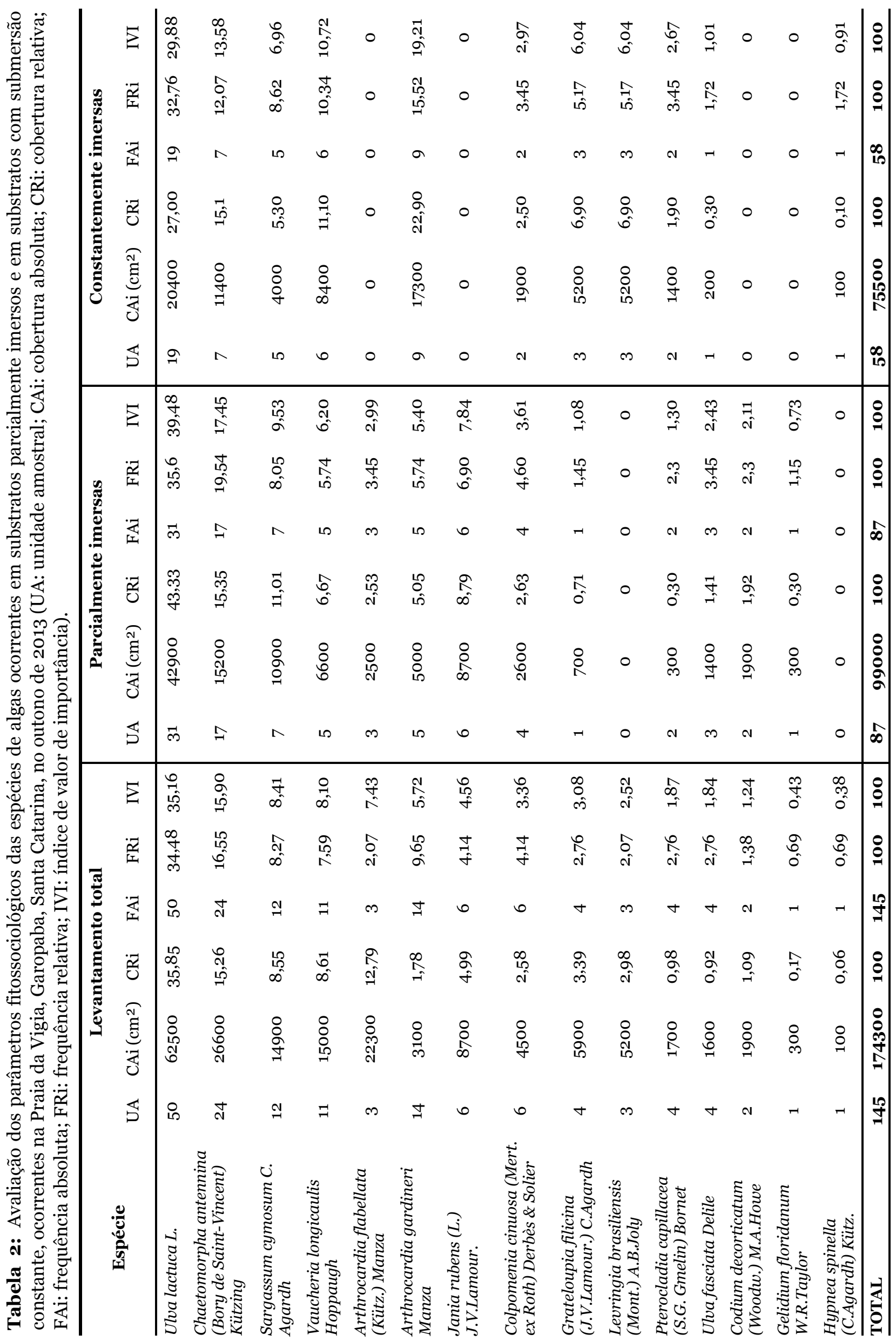


índice de equabilidade foi $\mathrm{J}=0,8 \mathrm{o}$, sendo o gênero Ulva, o mais abundante encontrado no estudo. Além disso, Ulva lactuca atingiu 41,1\% de abundância relativa, valores próximos aos obtidos no presente estudo.

Em outro estudo, Costa et al. (2012) avaliaram, entre outros parâmetros, a diversidade e equabilidade de comunidades de macroalgas bentônicas no litoral baiano, tendo sido analisadas 60 espécies, destas, 30 Rhodophyta e 20 Chlorophyta. Como parte dos resultados, obtiveram índices médios de $\mathrm{H}^{\prime}$ e $\mathrm{J}$ ' de 2,7 e 0,97, respectivamente. O gênero Ulva foi o mais frequente no estudo, ocorrendo em todos compartimentos recifais e em ambos os períodos de análise (seco e chuvoso), apresentando três espécies ( $U$. fasciata, $U$. rigida e $U$. lactu$c a)$.

Barbosa et al. (2008) citam U. fasciata como táxon dominante e mais frequente dentre as algas foliáceas, na região do mediolitoral de uma praia no Espírito Santo. Reis \& Yoneshigue-Valentin (1998), ao estudar a distribuição das macroalgas em uma laguna hipersalina do Rio de Janeiro, apontam Ulva como oportunista e tolerante a amplas variações de parâmetros ambientais. Isso justifica a presença de $U$. lactuca nas duas condições de inundação consideradas no presente estudo. Porém, houve redução dos valores de cobertura quando em constante inundação. Isso ocorreu com U. lactuca e Chaetomorpha antennina (Tabela 2), ambas do filo Chlorophyta, o que parece indicar que estas espécies conseguem sobreviver mais às condições de estresse hídrico, pois conseguem adaptar sua arquitetura, anatomia, metabolismo e crescimento como estratégia de sobrevivência, conforme afirma Righi (2012).

Em regiões mais eutrofizadas, espécies do gênero Ulva podem ser localmente dominantes, o mesmo ocorrendo em locais onde a salinidade é diminuída pelo aporte de água doce. Tal gênero, pertencente à família Ulvaceae, caracteriza-se por ser um grupo de algas verdes comestíveis que possui uma distribuição am- pla, ao longo das costas dos oceanos do planeta (Oliveira et al., 1999).

Existem registros de utilização dos gêneros Ulva e Enteromorpha como adubo por agricultores da região de Rio Grande (RS) (Oliveira Filho, 1981). Sugere-se assim, uma análise do papel ecológico desta biomassa algal nas praias brasileiras no intuito de exercer uma utilização sustentável do referido recurso, pois substâncias extraídas de macroalgas vêm apresentando bons resultados em ensaios anticancerígenos, antitrombóticos, antitumorais, antifúngicos e antivirais, sugerindo fonte potencial de novos fármacos (Oliveira, 1998). São poucos os estudos na costa catarinense. e estes abordam o potencial terapêutico das espécies encontradas

A avaliação de extratos de macroalgas bênticas do litoral catarinense pertencentes aos filos Chlorophyta, Phaeophyta e Rhodophyta em relação à atividade da larva Artemia salina, realizado por Lhullier et al. (2006), mostrou que as Rhodophyta apresentaram maior percentual de extratos com resultados estatisticamente mais significativos. Por fim, o trabalho publicado por Raymundo et al. (2004) descreve a atividade antioxidante in vitro de extratos de algumas algas verdes (Chlorophyta) ocorrentes no litoral catarinense. Isso salienta a importância de conhecer a diversidade de algas existentes na costa brasileira.

Cabe salientar ainda que, o estudo tratou de uma única atividade de pesquisa a campo, realizada nos dias 03 e 04 de maio de 2013, durante o outono. Dessa forma, os resultados são preliminares, em nível de prospecção inicial.

\section{CONCLUSÃO}

Os índices de diversidade obtidos se assemelham aos de outros estudos realizados na costa brasileira. Resultados semelhantes também foram registrados para o gênero Ulva, apontado em outros estudos como o mais a- 
bundante. Dentre as duas espécies registradas para o gênero, $U$. lactuca foi a mais frequente e apresentou os maiores valores de cobertura, independente da condição de inundação. Houve pouca variação entre as espécies e seus parâmetros fitossociológicos quando consideradas as diferenças de inundação constante ou não.

Dentre as espécies encontradas na amostragem analisada, não há registro novo para a costa catarinense. No entanto, o estudo contribui para uma prospecção geral da diversidade de algas macrófitas presentes na costa sul da Praia da Vigia, tendo em vista a existência de poucos estudos no litoral sul do Brasil.

\section{Referências Bibliográficas}

AMARAL, A. C. Z. \& S. JABLONSKI. 2005. Conservação da biodiversidade marinha e costeira no Brasil. Megadiversidade, 1: 43-51.

AMADO-FILHO, G. M., G. MANEVELDT, R. C. C. MANSO, B. V. MARINS-ROSA, M. R. PACHECO \& S. M. P. B. GUIMARÃES. 2007. Estructura de los mantos de rodolitos de 4 a 55 metros de profundidad em la costa sur del estado de Espírito Santo, Brasil. Ciéncias Marinas, 33: 1-12.

AMADO-FILHO, G. M., G. MANEVELDT, G. H. PEREIRA-FILHO, R. C. C. MANSO, R. G. BAHIA, M. B. BARROS-BARRETO \& S. M. P. B. GUIMARÃES. 2010. Seaweed diversity associated with a Brazilian tropical rhodolith bed. Ciéncias Marinas, 36: 371-391.

BAPTISTA, L. R. M. Flora Ilustrada do Rio Grande do Sul. Flora marinha de Torres (Chlorophyta, Xantophyta, Phaeophyta e Rhodophyta). Inst. Biociências. Fascículo XIV. Universidade Federal do Rio Grande do Sul, Porto Alegre, RS, 1977.

BARBOSA, S. O., M. A. O. FIGUEIREDO \& V. TESTA. 2008. Estrutura e dinâmica de comunidades bentônicas dominadas por macrófitas na zona intramareal da praia de Jacaraípe, Espírito Santo, Brasil. Hoehnea, 35: 563-575.

BLUNT, J., B. R. COPP, M. H. G. MUNRO, P. T. NORTHCOTE \& M. R. PRINSEP. 2005. Ma- rine natural products. Nat Prod Rep., 22: 1561.

COSTA, I. O., T. A. CAIRES, G. H PEREIRA FILHO \& J. M. C. NUNES. 2012. Macroalgas bentônicas associadas a bancos de Hypnea musciformis (Wulfen) J.V. Lamour. (Rhodophyta - Gigartinales) em duas praias do litoral baiano. Acta Bot. Bras., 26(2): 493507.

CONCENTINO, A. L. M., M. T. FUJI, N. V. REIS, N. C. GUIMARÃES-BARROS, M. F. ROCHA \& S. NEUMANN-LEITÃO. 2010. Diversity and distribution Patterns of the infralitoral green macroalgae from Potiguar basin, Rio Grande do Norte, Northeastern Brazil. Acta Bot. Bras., 24: 986-996.

FIGUEIREDO, M. A. O., M. B. B. BARRETO \& R. P. REIS. 2004. Caracterização das macroalgas nas comunidades marinhas da Área de proteção Ambiental de Cairuçú, Parati, RJ subsídios para futuros monitoramentos. Rev. Bras. Bot., 27: 11-17.

GHILARDI, N. P., G. H. PEREIRA FILHO \& F. BERCHEZ. 2008. Current knowledge status of the ecology of hard bottom benthic communities in brazil and the need for new approaches. Ecol. Bras., 12: 197-205.

HADLICH, R. M. 1984. Contribuição ao levantamento taxonômico das algas marinhas bentônicas do mangue do Itacorubi - Florianópolis - Ilha de Santa Catarina - Brasil - I Chlorophyta. Insula, 14.

HADLICH, R. M. \& Z. L. BOUZON. 1985. Contribuição ao levantamento taxonômico das algas marinhas bentônicas do mangue do Itacorubi - Florianópolis - Ilha de Santa Catarina - Brasil - II Rhodophyta. Insula, 15.

HAMMER, Ø., D. A. T. HARPER \& P. D. RYAN. 2001. PAST: Paleontological Statistics Software Package for Education and Data Analysis. Palaeo Electronica, 4(1): 1-9.

HORTA, P. A., E. AMÂNCIO, C. S. COIMBRA \& E. C. OLIVEIRA. 2001. Considerações sobre a distribuição e origem da flora de macroalgas marinha brasileiras. Hoehnea, 28: 243-265. 
JUDD, W. S., C. S. CAMPBEL, E. A. KELLONGG, P. F. STEENS \& M. J. DONOGUE. 2009. Sistemática Vegetal: um enfoque filogenético. 3. ed. Porto Alegre: Artmed, $612 \mathrm{p}$.

LHULLIER, C., P. A. HORTA \& M. FALKENBERG. 2006. Avaliação de extratos de macroalgas bênticas do litoral catarinense utilizando o teste de letalidade para Artemia salina. Rev. Bras. Farmacogn., 16(2): 158-163.

LITTLER, M., D. LITTLER, S. M. BLAIR \& J. N. NORRIS. 1985. Deepest known plant life discovered on an uncharted seamount. Science, 227: 57-59.

MACEDO, T. S., A. S. VARJÃO, L. L. FERNANDES, D. F. DA SILVA, J. S. J. C. ALMEIDA, A. S. DAS NEVES \& F. R. NONATO. 2009. Levantamento Taxonômico e Diversidade das Macroalgas Marinhas Bentônicas da Praia da Pituba, Salvador, Bahia. Rev. Elet. Biol., 2(2): 29-39.

MARTIN, L., K. SUGUIO, J. M. FLEXOR \& A. E. G. AZEVEDO. 1988. Mapa Geológico do Quaternário Costeiro dos Estados do Paraná e Santa Catarina. Série Geológica. Brasília.

MÜELLER-DOMBOIS, D. \& H. ELLENBERG. 1974. Aims and methods of vegetation ecology. New York: John Wiley, 45-66.

MUÑOZ, A. O. M. \& S. M. B. PEREIRA. 1997. Caracterização quali-quantitativa das comunidades de macroalgas nas formações recifais da praia do Cupe - Pernambuco, Brasil. Trabalho do Instituto Oceanográfico da Univiversidade Fedederal do Pernambuco, 25: 93-109.

NAGUIB, Y. M. A. 2000. Antioxidant activities of astaxanthin and related carotenoids. J Agric Food Chem., 48: 1150-1154.

NASSAR, C. 2012. Macroalgas Marinhas do Brasil: guia de campo das principais espécies. Rio de Janeiro: Technical Books Editora. 178 p.

NUNES, J. M. C., A. C. C. SANTOS, A. MINERVINO \& K. S. BRITO. 1999. Algas marinhas bentônicas do município de Ilhéus, Bahia, Brasil. Acta Bot Malacit., 24: 5-12.

ODUM, E. P. 1988. Ecologia. cap. 6. Rio de Janeiro: Guanabara Koogan S.A.
OLIVEIRA FILHO, E. C. A. 1981. Exploração de algas marinhas no Brasil: situação atual e perspectivas futuras. Phycological Latinoamerican, 1: 5-18.

OLIVEIRA, E. C. The seaweed resources of Brazil. 1998. In: CRITCHLEY, A. T. \& M. OHNO, Seaweed resources of the world. Yokosuka: Japan International Cooperation Agency, 367 $-371$.

OLIVEIRA, E. C., P. A. HORTA, C. E. AMANCIO \& C. L. SANT'ANNA. 1999. Avaliação de áreas prioritárias para conservação da biodiversidade da zona costeira e marinha. Algas e angiospermas marinhas bênticas do litoral brasileiro. Disponível em: < htpp://www.ib.usp./ algamare-br $>$ Acesso em: novembro de 2013.

OLIVEIRA, E. C., P. A. HORTA, C. E. AMANCIO \& C. L. SANT'ANNA. 2009. Algas e Angiospermas Marinhas Bênticas do Litoral Brasileiro: Diversidade, Exploração e Conservação. Instituto de Biociências da Universidade de São Paulo. São Paulo, 6o p. Disponível em: < http://www.anp.gov.br/brnd/round6/guias/ perfuracao/perfuracao r6/refere/ plantas marinhas.pdf $>$. Acesso em: maio de 2014.

PALUMBI, S. R. 2001. Marine Reserves and ocean neighboors: the spatial scale of marine populations and their management. Annu Rev Env Resour, 29: 31-68.

PEREIRA, S. M. B. 2002. Desenvolvimento e situação atual do conhecimento das macroalgas marinhas das regiões Nordeste e Norte. Pp. 117-121. In: ARAÚJO, E. L., A. N. MOURA, E. V. S. B. SAMPAIO, L. M. S. GESTINARI \& J. M. T. CARNEIRO. Biodiversidade, conservação e uso sustentável da flora do Brasil. Recife, Universidade Federal Rural de Pernambuco, Sociedade Botânica do Brasil, Seção Regional Pernambuco.

PREFEITURA MUNICIPAL DE GAROPABA. 2013. Disponível em: < http:// www.garopaba.sc.gov.br/conteudo/? $\underline{\mathrm{i}}$ $\underline{\text { tem }}=24436 \& \mathrm{fa}=3740 \&$ PHPSESSID $=\mathrm{q} 8$ esjmi aq5as251u4aj5cftmq2 > Acesso em: outubro 
de 2013.

RAVEN, P. H., R. F. EVERT \& S. E. EICHHORN. Biologia Vegetal. $7^{\mathrm{a}}$. ed. Editora Guanabara Koogan, Rio de Janeiro, 2007, p. 830.

RAYMUNDO, M. S., P. HORTA \& R. FETT. 2004. Atividade antioxidante in vitro de extratos de algumas algas verdes (Chlorophyta) do litoral catarinense (Brasil). Rev. Bras. Cienc. Farm., $40(4)$.

REIS, R. P., Y. YONESHIGUE-VALENTIN. 1998. Variação espaço-temporal de populações de Hypnea musciformis (Rhodophyta, Gigartinales) na baía de Sepetiba e armações dos Búzios, RJ, Brasil. Acta Bot. Bras., 12: 465483. (Suplemento)

RICKLEFS, R. E. 2003. A economia da Natureza. 5 ed. Rio de Janeiro: Guanabara Koogan S.A.

RIGHI, A. A., A. T. MATSUMURA, A. S. NUNES, B. N. T. DA SILVA, C. E. AMANCIO \& C. ILHA. 2012. Botânica de Inverno. Instituto de Biociências da Universidade de São Paulo, 183.

RIUL, P., P. LACCOUTH, P. R. PAGLIOSA, M. L. CHRISTOFFERSEN \& P. A. HORTA. 2009. Rhodolith beds at the easternmost extreme of South America: Community structure of an endangered environment. Aquatic Bot., 90: 315-320.

SEELIGER, U., C. ODEBRECHT \& J. P. CASTELLO. 1998. Os Ecossistemas Costeiro e Marinho do Extremo Sul do Brasil. Rio Grande, RS. Ecoscientia, 362 p.

VILLAÇA, R. \& F. B. PITOMBO. 1997. Benthic communities of shallow-water reefs of Abrolhos, Brazil. Rev. Bras. Oceanogr., 45: 35-43.

VILLAÇA, R., A. C. FONSECA, V. K. JENSEN \& B. KNOPPERS. 2010. Species composition and distribution of macroalgae on Atol das Rocas, Brazil, SW Atlantic. Bot Mar., 53: 113122.

WALKER, D. I. \& G. A. KENDRICK. 1998. Threats to macroalgal diversity: Marine habitat destruction and fragmentation, pollution and introduced species. Bot Mar., 41: 105112. 